\title{
Bending modes, elastic constants and mechanical stability of graphitic systems
}

\author{
G. Savini,,${ }^{1,2}$ Y. J. Dappe, ${ }^{3}$ S. Öberg, ${ }^{4}$ J. -C. Charlier,${ }^{5}$ M. I. Katsnelson, ${ }^{1}$ and A. Fasolino ${ }^{1}$ \\ 1 Institute for Molecules and Materials, Radboud University Nijmegen, 6525ED Nijmegen, The Netherlands \\ ${ }^{2}$ Department of Engineering, University of Cambridge, CB3 OFA Cambridge, United Kingdom \\ ${ }^{3}$ Institut de Physique et Chimie des Matériaux, CNRS, F-67034 Strasbourg, France \\ 4 Department of Mathematics, Lulea University of Technology, S-97187 Luleå, Sweden \\ ${ }^{5}$ Institute of Condensed Matter and Nanosciences (IMCN), Université Catholique de Louvain, \\ Place Croix du Sud 1 (NAPS-Boltzmann), B-1348 Louvain-la-Neuve, Belgium
}

\begin{abstract}
The thermodynamic and mechanical properties of graphitic systems are strongly dependent on the shear elastic constant $C_{44}$. Using state-of-the-art density functional calculations, we provide the first complete determination of their elastic constants and exfoliation energies. We show that stacking misorientations lead to a severe lowering of $C_{44}$ of at least one order of magnitude. The lower exfoliation energy and the lower $C_{44}$ (more bending modes) suggest that flakes with random stacking should be easier to exfoliate than the ones with perfect or rhombohedral stacking. We also predict ultralow friction behaviour in turbostratic graphitic systems.
\end{abstract}

\section{INTRODUCTION}

Graphitic systems are used for a wide variety of industrial applications, ranging from lubricant and refractory materials to neutron moderators in nuclear fission reactors ${ }^{1}$ and plasma shields in the next generation of

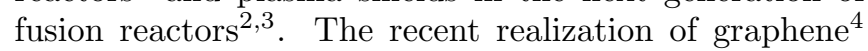
(single graphitic layer) and the discovery of its unusual electronic properties ${ }^{566}$ have raised the interest on flake graphitic systems as a route to produce graphene samples of high quality and in large scale ${ }^{7 / 9}$.

Despite the technological and scientific importance of graphitic systems, the knowledge of their elastic properties is unexpectedly poor and new insights are needed. The values of the elastic constants describe the mechanical behaviour ${ }^{10}$ and are decisive in engineering design to avoid material failure. In layered materials, they are even more important for the thermodynamic properties due to a low-lying branch of acoustic vibrations, the bending modes, predicted by Lifshitz ${ }^{11}$ over fifty years ago. Here we show that the shear elastic constant $C_{44}$ affects the mechanism of exfoliation that is relevant for the production of graphene.

The most reliable experimental studies of the elastic constants have been obtained by inelastic x-ray scattering ${ }^{12}$ and ultrasonic, sonic resonance, and static test methods $\frac{13}{}$. The sample used in the first study $\underline{12}$ was single-crystalline Kish graphite, characterized by an extraordinary high degree of ordering, the closest approximation to the perfect $\mathrm{AB}$ stacking graphite (hex-g). The second study ${ }^{13}$ was done using highly oriented pyrolitic graphite, the closest approximation to turbostratic graphite (turbo-g) where the graphitic layers are randomly oriented around the c-axis.

Except for $C_{44}$ and $C_{13}$, both studies are in agreement within the experimental uncertainties. The $C_{13}$ value in turbo-g was determined only by the less accurate static test method and it may be affected by errors. Conversely, $C_{44}$ in turbo-g was determined from the sound velocity and its value ranges between $0.18-0.35 \mathrm{GPa}^{13}$, one order of magnitude lower than $5.03 \mathrm{GPa}$ found in hex-g

The discrepancy on $C_{44}$ is attributed to the existence of mobile basal dislocations 13114 . After neutron irradiation, the elastic constant $C_{44}$ increases by up to an order of magnitude ${ }^{14}$, suggesting that interstitial defects ${ }^{15}$ could pin the dislocation motions, whence the intrinsic value of $C_{44}$ is measured. A principal difficulty with this explanation is that interstitial atoms inevitably increase the shear resistance between graphitic layers and therefore they may increase the $C_{44}$ value by themselves.

The aim of this study is to investigate from first principles the elastic constants of graphitic systems with respect to the stacking misorientations between layers, and to describe the key role of the shear elastic constant $C_{44}$ on the bending modes (thermal property) and mechanical stability. We show that stacking misorientations greatly affect $C_{44}$ and that graphitic systems with perfect (hexg) and random (turbo-g) stacking should be considered as two distinct materials described by their own elastic and thermodynamic properties.

This paper is structured as follow. In Sec. II] we give a brief summary of the theoretical methods and a discussion of the LCAO-S ${ }^{2}+\mathrm{vdW}$ formalism to include the long-range van der Waals (vdW) interactions. The consequences of shear elastic constant $C_{44}$ on the bending modes and mechanical stability are shown in Sec. III A and IIIB, respectively.

The elastic constants in the case of high-symmetric systems (hexagonal, orthorhombic, rhombohedral and AA hexagonal stackings) and for graphitic layers randomly oriented around the c-axis (turbostratic stacking) are presented in Sec. IIIC and IIID, respectively. Finally, we summarize and comment on our results (Sec. IV).

\section{METHOD}

All the calculations are performed using density functional theory, within the local density approximation scheme (LDA), norm-conserving pseudopotentials ${ }^{16}$ and plane waves with cut-off energy of 150 Ry (ABINIT 
package $)^{17}$. The k-point mesh was chosen so that the average density corresponds approximately to a $32 \times 32 \times 16$ mesh for hex-g. Energies were converged within 0.05 $\mathrm{meV} /$ atom, and elastic constants within $0.5 \%$. For large supercells (turbo-g with more than 50 atoms) we have used localized basis-set composed of Gaussian orbitals (AIMPRO code) 18 . The elastic constants calculated by the two LDA codes are in agreement within $3 \%$ or better.

The choice of LDA is not fortuitous ${ }^{19}$ and it was dictated by test calculations using the generalizedgradient approximation (GGA) within the PerdewBurke-Ernzerhof scheme ${ }^{20}$. According to GGA, the distance between graphitic layers is far too large $(4.2 \AA)$, resulting in a negligible interlayer binding energy and almost vanishing out-of-plane elastic constants $\left(C_{44}, C_{33}\right)$. For these reasons we have dismissed the use of GGA from this study 2123 .

Even though LDA yields accurate equilibrium distances, due to energetical error compensations, the longrange van der Waals (vdW) and more generally the weak contribution to the out-of-plane interactions is not well described $^{24 \mid 25}$. In order to check the importance of these effects on $C_{44}$ and $C_{33}$, we have used the LCAO-S ${ }^{2}+\mathrm{vdW}$ formalism to include these specific interactions within LDA $^{26}$ (using FIREBALL code) 2 .

This formalism takes into account two major contributions. The first one, which we call weak chemical interaction, is a repulsive energy originating from the overlaps of electronic densities between the weakly interacting subsystems. Even though the overlaps are rather small, this energy is not negligible. This contribution is evaluated proceeding to a second order expansion of the electronic wavefunctions with respect to the overlaps.

The second contribution, which is the vdW itself, originating from charge fluctuations, can be seen as the interaction between electronic dipoles. In the frame of the dipolar approximation, we use a second order perturbation theory to describe this contribution. This method has originally been tested with success on standard graphene-graphene interaction, and more recently on a wide range of graphitic materials 28 . In its current stage, the analysis of the internal stresses is not implemented yet, thus the elastic constants that change the in-plane bond lengths $\left(C_{11}, C_{12}, C_{13}\right)$ are overestimated by up to $15 \%$. However, for $C_{44}$ and $C_{33}$ that describe the weak interaction between layers, the internal stresses are negligible, and the calculated values are expected to be very accurate.

The elastic constants are determined using two different approaches. The first one uses the response-function, implemented in the ABINIT code, to calculate the second derivative of the total energy with respect to the strains.

The second approach uses the elastic energy density 29 . For each elastic constant we have applied 21 strain components $\varepsilon_{i j}$ to the equilibrium crystal structures $\left(\varepsilon_{i j}\right.$ were typically ranging between 0.01 with increments 0.001 ). The elastic constants are found by fitting the calculated energies to a polynomial function in the strains. Both
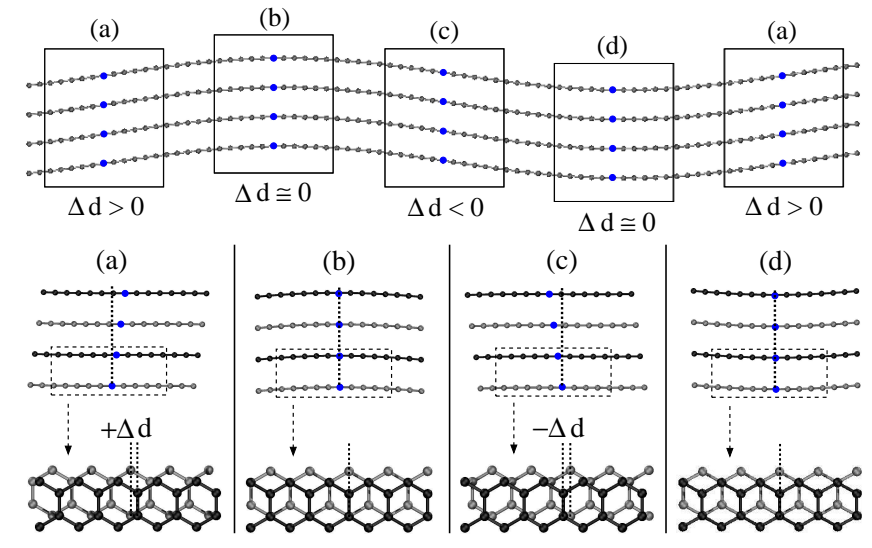

FIG. 1: (Color online) Transversal acoustic (bending) mode. The bending changes the local stacking between graphitic layers. The boxes (a-d) show regions with different slopes and stackings. The shear $\triangle d$ gives the deviations from $\mathrm{AB}$ phase (perfect stacking).

approaches yield results in agreement within $0.2 \%$.

\section{RESULTS AND DISCUSSION}

\section{A. Bending modes}

The bending modes are atomic vibrations that can be excited even at low temperature and strongly influence the thermal properties of layered materials. In 1952, Lifshit $2^{11}$ obtained the following dispersion law for the out-of-plane acoustic mode $\omega$ :

$$
\rho \times \omega^{2}(q)=C_{44}\left(q_{x}^{2}+q_{y}^{2}\right)+C_{33} q_{z}^{2}+\kappa\left(q_{x}^{2}+q_{y}^{2}\right)^{2} / c
$$

where $\rho$ is the density, $c$ is the interlayer distance, $q_{x, y, z}$ are the wave vectors and $\kappa$ describes the intralayer forces characterizing the bending rigidity $(1.1 \mathrm{eV})^{30}$.

The small value of $C_{44}$ (characteristic of graphitic systems, see Table II leads to a predominant contribution of the transversal bending modes $\left.\left(q_{z}=0\right)\right)$ in the phonon dispersion curves. These modes are sinusoidal displacements that propagate along the planes and change the local stacking between layers (see Fig. 1). For nearly flat planes the shear stacking $\Delta d$ is almost zero, whereas, for positive (or negative) slope $\triangle d$ becomes positive (or negative). Using trigonometric considerations, it can be shown that the maximum value of $\triangle d$ (see boxes in Fig. 1) is given by:

$$
\triangle d=\frac{\pi \widehat{a} c}{\lambda} \times \frac{1}{\sqrt{1+\left(\frac{2 \pi \cdot \widehat{a}}{\lambda}\right)^{2}}}
$$

where $\widehat{a}$ is the amplitude, $\lambda$ is the wavelength and $c$ is the interlayer distance.

The crystal resistance to the stacking shear ${ }^{29}$ is proportional to $E \propto C_{44} \times \triangle d^{2}$, and by lowering $C_{44}$ more 
bending modes can be excited at lower temperature. In the limit of $C_{44}=0$, graphitic systems approach the graphene behaviour, where indeed bending modes (or ripples) are always present 30 .

\section{B. Mechanical stability}

By imposing the elastic strain energy as positively definite $^{29}$, the stability conditions are given by:

$$
2 C_{13}^{2}<C_{33}\left(C_{11}+C_{12}\right) \quad C_{11}, C_{12}, C_{33}, C_{44}>0
$$

Note that $C_{13}$ does not affect the stability: a positive (or negative) value means that under in-plane compression the out-of-plane distance tends to expand (or contract).

The elastic constants $C_{11}, C_{12}$ describe in-plane deformations and they possess the highest values due to the strong $\mathrm{sp}^{2}$ bonding interactions within the graphene planes. The elastic constant $C_{33}$ describes out-of-plane compression or expansion and it has always a positive value for perfect and stacking misorientations (see Table I).

The elastic constant $C_{44}$ corresponds to a shear between graphene layers. Due to the weak interaction between planes, the $C_{44}$ value is the lowest and can be positive or negative depending on the stacking misorientations (see Table I). The latter elastic constant is the only one that can break the mechanical stability condition (i.e. $\left.C_{44}<0\right)$.

\section{Elastic constants in high-symmetric graphitic systems}

By imposing a translation vector between graphitic layers we found four high-symmetric stackings that correspond to stationary points on the stacking-fault energy surface ${ }^{31}$. To calculate the stacking-fault energy surface (for graphitic system is often called corrugation energy surface $)^{32 / 34}$, we have used 16-atoms unit cell model of eight layers in $\mathrm{AB}$ sequence in which the stacking at the unit cell boundary is changed by imposing a shear displacement. This represents an intrinsic stacking fault between layers at the unit cell boundaries whereas the others remains stacked in the $\mathrm{AB}$ sequence. The multiple layers repeat along the c-axis (8 layers) makes negligible self-interaction of the intrinsic fault ${ }^{31}$. The stationary points, indicated with square and circle symbols in Fig. 2 a, correspond to the four high symmetric structures (hexagonal, rhombohedral, orthorhombic and AA hexagonal graphite, see Fig. 2p). The in-plane lattice parameter was $2.45 \AA$ very close to the experimental value $2.463 \AA^{12}$, with no significant changes among all the graphitic structures.

The origin in Fig. 2a corresponds to hex-g (black square symbol), the global minimum of the energy surface with an interlayer separation of $3.34 \AA$ (experimental value $3.356 \AA)^{12}$. With the exception of the $C_{33}$ value (29 (a)

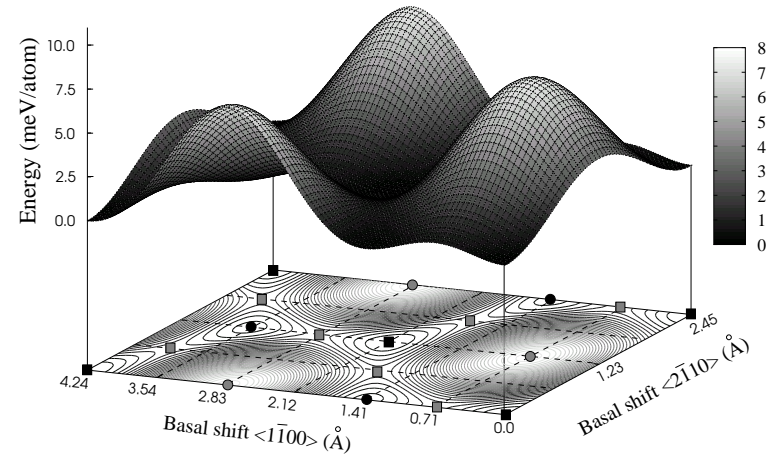

(b)

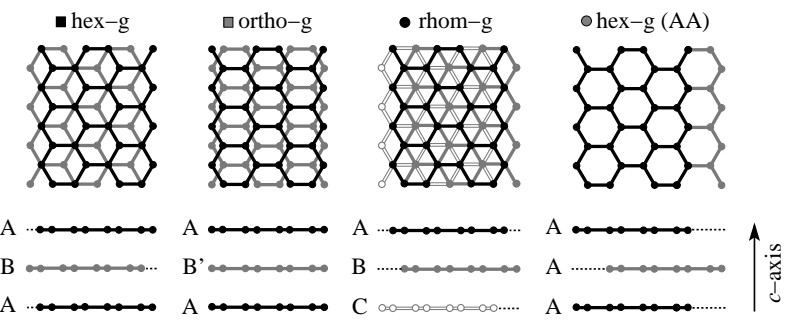

FIG. 2: (a) Stacking-fault energy surface (also called corrugation energy surface). The square and circle symbols indicate the stationary points corresponding to the following high-symmetric structures; (b) The hexagonal, orthorhombic, rhombohedral and AA hexagonal stackings viewed perpendicular (above), parallel (below) to the c-axis. The energies of these structures with respect to hex-g are $1.66 \mathrm{meV} /$ atom for ortho-g, $0.10 \mathrm{meV} /$ atom for the rhom-g and $9.29 \mathrm{meV} /$ atom for AA hex-g (see text).

GPa), the calculated elastic constants (given in Table I) are in agreement within the experimental uncertainties found in hex-g $\mathrm{g}^{12}$. Using the LCAO- $\mathrm{S}^{2}+\mathrm{vdW}$ formalism, the out-of-plane elastic constant $C_{33}$ becomes $42 \mathrm{GPa}$, very close to the experimental value $38.7 \pm 7 \mathrm{GPa}^{12}$.

The local minimum at $1 / 3\langle 1 \overline{1} 00\rangle$ (black circle symbol) represents the rhombohedral stacking (rhombo-g). This structure possesses the same interlayer separation and elastic constants of hex-g (the differences are beyond the accuracy of the calculations) with formation energy of $0.10 \mathrm{meV} /$ atom. This very small energy explains why the rhombohedral phase is usually $5-15 \%$ intermixed with the perfect hexagonal one in natural graphitic flakes.

The saddle point at $1 / 6\langle 1 \overline{1} 00\rangle$ (grey square symbol) represents the orthorhombic stacking (ortho-g). The interlayer separation of the primitive unit cell is $3.37 \AA$ with formation energy of $1.66 \mathrm{meV}$ /atom. The latter energy represents the lowest barrier that has to be overcome during the shearing process from an ideal configuration to another equivalent one. The $C_{44}$ values are found to range between $-2.7 \mathrm{GPa}$, for shearing along $\langle 1 \overline{1} 00\rangle$, and 7.7 GPa, for shearing along the $\langle 2 \overline{1} 10\rangle$ axis. No significant changes are found for the other elastic constants (see Table I). Although unstable (due to the negative $C_{44}$ ), this structure acts as an intermediate phase during the transformation from graphite to diamond 35 .

The global maximum at $2 / 3\langle 1 \overline{1} 00\rangle$ (grey circle symbol) 


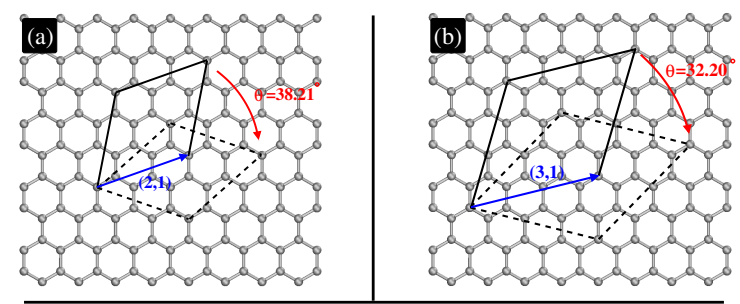

(c)

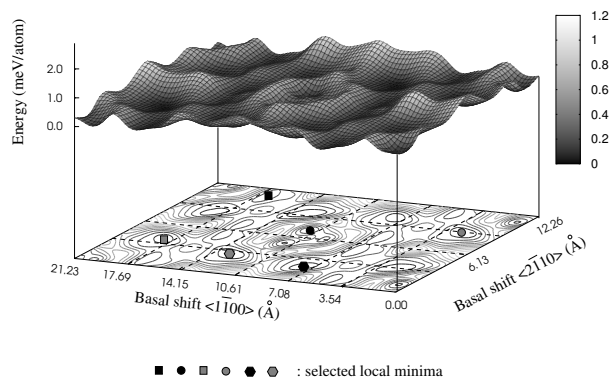

FIG. 3: (Color online) (a,b) Representation of accidental commensuration. A supercell of vector $(n, m)$ (blue colour) becomes commensurate when rotated by an angle $\theta$ with respect to the starting supercell (as we increase $n, m$ the supercell surface and number of atoms rapidly increase). (c) Stacking fault energy surface (or corrugation energy surface) of a graphene bilayer with supercell vector $(2,1)$. The respective elastic constants are calculated on the local minima of the energy surface. Notice that the energy surface becomes much flatter leading to a reduction of $C_{44}$.

corresponds to AA hexagonal stacking (AA hex-g). This phase has the largest interlayer separation (3.60 $\AA$ ) and the highest formation energy $(9.29 \mathrm{meV} /$ atom $)$. Its elastic constants are smaller than in hex-g, and $C_{44}$ in particular, becomes negative $(-3.8 \mathrm{GPa})$ breaking the stability conditions. Even though this structure is highly unstable, a recent study has suggested that screw dislocations locally encourage this stacking .

In the following section we describe the elastic constants for graphitic layers randomly oriented around the c-axis.

\section{Elastic constants in turbostratic stacking}

The modelling of turbostratic staking is challenging since the incommensurate nature of these stackings must combine with the finite-size constraint required by calculations. To overcome this difficulty we used the method proposed by Kolmogorov and Crespi $\sqrt{32}$ in which a layer with supercell basis vector $(n, m)$ becomes commensurate with a second layer for a specific rotation angle of:

$$
\vartheta=\cos ^{-1}\left[\frac{2 n^{2}+2 n m-m^{2}}{2\left(n^{2}+n m+m^{2}\right)}\right] \quad \text { with } n>m
$$

Figure 3 a,b shows the case of the two smaller supercell with basis vector $(2,1)$ and $(3,1)$ (corresponding to 14,26 atoms/layer and rotation angles of $38.21^{\circ}, 32.20^{\circ}$,
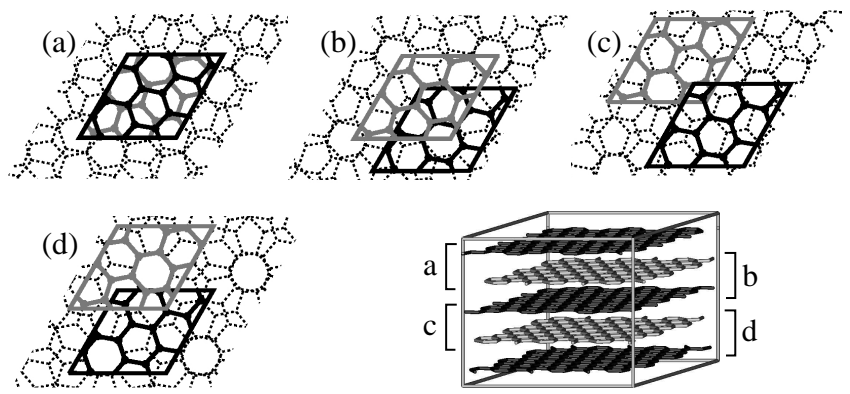

FIG. 4: An example of 5-layers turbostratic stacking (supercell basis vector 2,1). Each layer is stacked along the c-axis, rotated with respect to each other with an angle of $38.21^{\circ}$ and randomly translated along the basal plane.

respectively). Figure 3. shows the stacking energy surface for a bilayer supercell of basis vector $(2,1)$. To better describe the complete misorientation of turbostratic stacking we have increased the number of layers along the c-axis. Each layer was rotated with respect to each other and randomly translated along the basal plane (see Fig. (4). The rotation angles used are 15 values ranging from $6.01^{\circ}$ to $53.99^{\circ}$. The smallest supercell contains 28 atoms with 2 layers rotated with respect to each other by $38.21^{\circ}$, whereas the largest one contains 456 atoms with 12 layers and rotation angle of $46.83^{\circ}$.

For each model we carried out extensive structural optimizations starting from different translation vectors along the basal plane. The corrugation energy is about one order of magnitude lower than commensurate structure (see Fig. 2a) with a maximum value of $0.9 \mathrm{meV} /$ atom (see Fig. 35). As we increased the size of the supercell over the basal plane we found that the average corrugation energy tends to decrease up to $20 \%$ for the largest size (basis vector 8,3 with 194 atoms/layer). Extrapolating these results in the ideal case of infinite layers, we suggest corrugation energy virtually flat with layers mutual independent.

For all the supercell studied the in-plane lattice parameters remain almost equal to the value found in hex-g, whereas the interlayer distances were on average slightly larger, $3.42 \pm 0.01 \AA$, with formations energies of $3.03 \pm 0.6$ $\mathrm{meV} /$ atom.

The interlayer binding energy between graphitic layers (i.e. exfoliation energy) was $21 \pm 1 \mathrm{meV} /$ atom $(70 \pm 4$ $\mathrm{meV}$ /atom with vdW), a value slightly lower than the $24 \mathrm{meV} /$ atom found in hex-g $(80 \mathrm{meV} /$ atom with vdW). Note that LDA values yield to a binding energy within a factor of $2-3$ with respect to $\mathrm{LCAO}^{2} \mathrm{~S}^{2}+\mathrm{vdW}$ formalism and experiment values ( $43 \mathrm{meV} /$ atom found in heat-ofwetting experiment 37 , $35 \pm 10 \mathrm{meV} /$ atom found by analyzing TEM images of twisted collapsed nanotubes 38 , and $52 \pm 5 \mathrm{meV} /$ atom by studying thermal desorption of polyaromatic hydrocarbons $\left.{ }^{39}\right)$. In Table I we report the calculated values of the elastic constants. With the exception of $C_{44}$, these values hardly change among all the studied turbostratic stackings with no clear dependence 


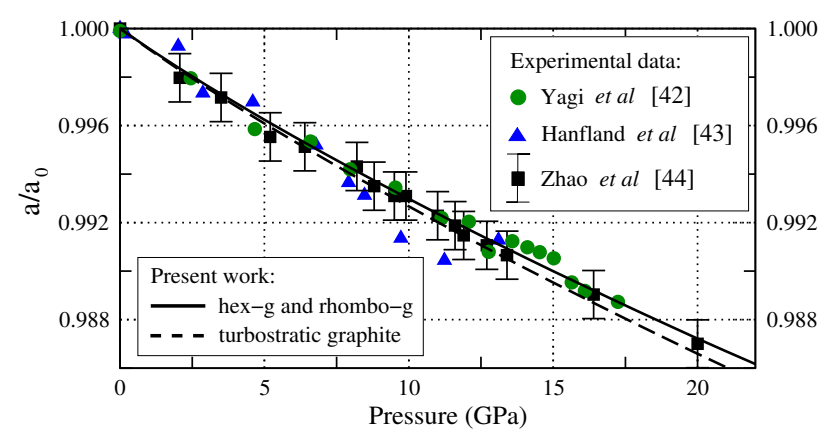

FIG. 5: (Color online) In-plane lattice parameters vs. pressure. The solid line represents the results found for hex-g and rhombo-g (perfect and rhombohedral stackings). The dashed line shows the results found here for turbostratic graphite. For comparison, the experimental results are also plotted.

on the rotation angles and number of layers. The elastic constants $C_{11}, C_{12}$ slightly decrease by about $3 \%$ with respect to hex-g, remaining within the experimental uncertainties found in turbostratic samples 13 . As previously found in hex-g, LDA calculations underestimate the $C_{33}$ value $(27 \pm 2 \mathrm{GPa})$ with respect to the vdW correction $(36 \pm 1 \mathrm{GPa})$ and the experimental value of $36.5 \pm 1 \mathrm{GP} \mathrm{a}^{13}$.

Conversely, we found $C_{13}=-2.7 \pm 0.5 \mathrm{GPa}$ in disagreement with the experimental value $15 \pm 5 \mathrm{GPa} 13$. The latter value was only indirectly obtained as a function of the other elastic constants by the less accurate static test method. This method requires larger strains than ultrasonic experiments and non-linear behaviour of stressstrain curve may affect the measured value. Furthermore, the linear bulk modulus $B_{a}$ calculated from these elastic constants is far too large (2080 GPa), almost double than the one found in diamond $(1326 \mathrm{GPa}) 40$.

The linear bulk modulus $B_{a}$ describes the variation of the lattice parameter $a$ as a function of the hydrostatic pressure 41 and it is given by:

$$
B_{a}=\frac{C_{33}\left(C_{11}+C_{12}\right)-2 C_{13}^{2}}{C_{33}-C_{13}}
$$

This modulus is strongly weighted by $C_{13}$. For example, if we use the measured value found in hex-g $0 \pm 3 \mathrm{GPa}$ (instead of $15 \pm 5 \mathrm{GPa}$ ), $B_{a}$ becomes $1240 \mathrm{GPa}$ (instead of $2080 \mathrm{GPa}$ ).

Several x-ray studies have measured the linear bulk modulus $B_{a}$. In these experiments powder samples were prepared by grinding different types of graphitic materials, ranging from well crystallized to poorly crystallized grains 4244 . The good agreement between the experiments (see Fig. 5 indicates that the linear bulk modulus $B_{a}$ does not strongly depend on the stacking order with a measured value of $1250 \pm 70 \mathrm{GPa}{ }^{43}$. By including the latter modulus in the Eq. 5, the elastic constant $C_{13}$ becomes $0.3 \mathrm{GPa}$. Therefore, we conclude that the $C_{13}$ value does not significantly change between turbo-g and hex-g and we propose that the same value $0 \pm 3 \mathrm{GPa}$ should be appropriate also for turbostratic stacking.

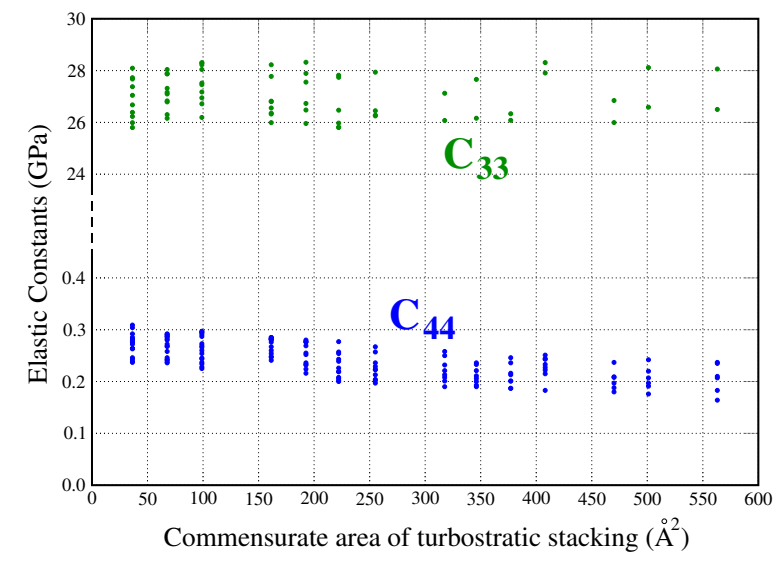

FIG. 6: (Color online) The elastic constants $C_{44}$ and $C_{33}$ as a function of the commensurate area over the basal plane in turbostratic stacking. The $C_{44}$ value tends to decrease with respect to the supercell size, whereas $C_{33}$ is not quantitatively affected.

The $C_{44}$ represents the second derivative of the total energy as a function of shear displacement over the basal plane. As the corrugation energy of perfect $\mathrm{AB}$ stacking is much higher than turbostratic we are expecting a corresponding lower value of the shear elastic constant. We have found that $C_{44}$ tends to decrease as a function of the supercell area over the basal plane (see Fig. 6) and is independent on the relative rotation angles and number of layers along the c-axis (the respective average corrugation energies and therefore the $C_{44}$ values are also independent on the relative rotation angles and number of layers).

For commensurate structures of finite area ranging between $36-563 \AA^{2}$ the respective $C_{44}$ values are within 0.16-0.31 GPa, one order of magnitude lower than hex-g $(4.5 \mathrm{GPa})$ and close to the experimental measures ranging from 0.18 to $0.35 \mathrm{GPa}^{13}$. The $\mathrm{vdW}$ correction does not significantly affect the $C_{44}$ values with respect to LDA (see Table I), suggesting that the variations in energy under interlayer shears are nearly identical between these two approximations. As we increase the sizes of the commensurate structures towards the ideal case of infinite layers (incommensurate) the $C_{44}$ values tend to zero with corrugation energy virtually flat. These results indicate that turbostratic stacking possesses the lowest friction among all the graphitic materials bearing great potential applications in nano-mechanical systems $\$ 5146$.

\section{CONCLUSIONS}

To summarize, we have discussed the importance of $C_{44}$ as the main parameter that restrains the bending modes and controls the mechanical stability of layered materials. Using advanced ab-initio method, which includes vdW interactions, we have provided the first complete description of the elastic constants in graphitic sys- 
TABLE I: Elastic constants in unit of GPa for the different graphitic systems. The values between brackets are calculated using the LCAO-S ${ }^{2}+\mathrm{vdW}$ formalism. These results show that the $C_{13}$ values do not significantly change between turbo-g and hex-g and we propose that the same value $0 \pm 3 \mathrm{GPa}$ should be appropriate also for turbostratic stacking. The shear elastic constants $C_{44}$ found in turbostratic stacking correspond to commensurate structures of area ranging between $36-563 \AA^{2}$.

\begin{tabular}{|c|c|c|c|c|c|c|c|}
\hline & \multicolumn{2}{|c|}{ hex-g (AB) } & \multicolumn{2}{|r|}{ turbo-g } & rhombo-g & \multirow{2}{*}{$\begin{array}{l}\text { ortho-g } \\
\text { Theory }\end{array}$} & \multirow{2}{*}{$\frac{\text { hex-g (AA) }}{\text { Theory }}$} \\
\hline & Experiment 12 ] & Theory & Experiment 13 & Theory & Theory & & \\
\hline$C_{11}$ & $1109 \pm 16$ & 1109 & $1060 \pm 20$ & $1080 \pm 3$ & 1107 & 1095 & 1028 \\
\hline$C_{12}$ & $139 \pm 36$ & 175 & $180 \pm 20$ & $171 \pm 4$ & 175 & 173 & 162 \\
\hline$C_{33}$ & $38.7 \pm 7$ & $29(42)$ & $36.5 \pm 1$ & $27 \pm 2(36 \pm 1)$ & $29(42)$ & $26(38)$ & $21(30)$ \\
\hline$C_{13}$ & $0 \pm 3$ & -2.5 & $15 \pm 5$ & $-2.7 \pm 1$ & -2.5 & -2.6 & -3.0 \\
\hline$C_{44}$ & $5.0 \pm 3.0$ & $4.5(4.8)$ & $0.18 / 0.35$ & $0.16 / 0.31(0.19 / 0.34)$ & $4.4(4.8)$ & $-2.7 / 7.7(-2.9 / 7.3)$ & $-3.8(-3.8)$ \\
\hline
\end{tabular}

tems. The lower exfoliation energy (3-8 meV/atom) and the lower $C_{44}$ (at least one order of magnitude) found in turbostratic stacking suggest that the exfoliation mechanism, relevant for the production of graphene, should be easier for graphite flakes with random stacking. Our results indicate that turbostratic graphitic systems possess the lowest friction among all the graphitic stackings. It would be interesting to check these predictions experimentally.

\section{Acknowledgments}

The authors thank the HPC-EUROPA project for financial and computer supports. Computational re- sources have also been provided by the Université Catholique de Louvain on the LEMAITRE and GREEN computers of the CISM. J.-C.C. acknowledges financial support from the F.R.S.-FNRS of Belgium; G.S. from JSPS and Grant-in-Aid for Scientific Research.
1 Klimenkov VI. Behavior of graphite in nuclear reactor stacks. Atom. Energy 1962; 10(5):439-450.

2 Evans TE, Moyer RA, Burrell KH, Fenstermacher ME, Joseph I, Leonard AW, et al. Edge stability and transport control with resonant magnetic perturbations in collisionless tokamak plasmas. Nature Phys. 2006; 2:419-423.

3 Scaffidi-Argentina F, Safronov V, Arkhipov I, Arkhipov N, Bakhtin V, Barsuk V, et al. Erosion mechanisms and products in graphite targets under simulated disruption conditions. J. Nucl. Mater. 2000;283-287(2):1111-1115.

${ }^{4}$ Novoselov KS, Geim AK, Morozov SV, Jiang D, Zhang Y, Dubonos SV, et al. Electric Field Effect in Atomically Thin Carbon Films. Science 2004;306(5696):666-669.

${ }^{5}$ Novoselov KS, Geim AK, Morozov SV, Jiang D, Katsnelson MI, Grigorieva IV, et al. Two-dimensional gas of massless Dirac fermions in graphene. Nature 2005;438: 197-200.

6 Zhang Y, Tan Y, Stormer HL, Kim P. Experimental observation of the quantum Hall effect and Berry's phase in graphene. Nature 2005;438:201-204.

7 Li X, Wang X, Zhang L, Lee S, Dai H. Chemically Derived, Ultrasmooth Graphene Nanoribbon Semiconductors. Science 2008;319:1229-1232.

8 Hernandez Y, Nicolosi V, Lotya M, Blighe FM, Sun Z, De $\mathrm{S}$, et al. High-yield production of graphene by liquid-phase exfoliation of graphite. Nature Nanotech. 2008;3:563-568.

9 Siegel DA, Hwang CG, Fedorov AV, Lanzara A. Quasifreestanding multilayer graphene films on the carbon face of SiC. Phys. Rev. B 2010;81:241417.

10 Mohiuddin TMG, Lombardo A, Nair RR, Bonetti A,
Savini G, Jalil $\mathrm{R}$ et al. Uniaxial strain in graphene by Raman spectroscopy: $G$ peak splitting, Grüneisen parameters, and sample orientation. Phys. Rev. B 2009;79(20):205433.

11 Lifshitz IM. On the thermal properties of chain and layered structures at low temperatures. Zh. Eks. Teor. Fiz. 1952;22(4):475-486.

12 Bosak A, Krisch M, Mohr M, Maultzsch J, Thomsen C. Elasticity of single-crystalline graphite: Inelastic $\mathrm{x}$-ray scattering study. Phys. Rev. B. 2007;75(15):153408.

13 Blakslee OL, Proctor DG, Seldin EJ, Spence GB, Weng T. Elastic Constants of Compression-Annealed Pyrolytic Graphite. J. Appl. Phys. 1979;41(8):3373.

14 Seldin EJ, Nezbeda CW, Elastic Constants and Electron-Microscope Observations of Neutron-Irradiated Compression-Annealed Pyrolytic and Single-Crystal Graphite. J. Appl. Phys. 1970;41(8):3389.

15 Suarez-Martinez I, El-Barbary AA, Savini G, Heggie MI. First-Principles Simulations of Boron Diffusion in Graphite. Phys. Rev. Lett. 2007;98(1):015501.

16 Troullier N, Martins JL. Efficient pseudopotentials for plane-wave calculations. II. Operators for fast iterative diagonalization. Phys. Rev. B 1991;43(11):8861-8869.

17 Gonze X, Beuken J-M, Caracas R, Detraux F, Fuchs M, Rignanese G-M, et al. First-principles computation of material properties: the ABINIT software project. Comput. Mater. Sci. 2002;25(3):478-492.

18 Briddon PR, Jones R. LDA Calculations Using a Basis of Gaussian Orbital. Phys. Stat. Sol. (b) 2000;217(1):131-171. 
19 Perdew JP, Zunger A. Self-interaction correction to density-functional approximations for many-electron systems. Phys. Rev. B 1981;23(10):5048-5079.

${ }^{20}$ Perdew JP, Burke K, Ernzerhof M. Generalized Gradient Approximation Made Simple. Phys. Rev. Lett. 1996;77(18):3865-3868.

21 Tournus F, Charlier J-C, Melinon P. Mutual orientation of two C60 molecules: An ab initio study. J. Chem. Phys. 2005;122(9):094315.

${ }^{22}$ Charlier J-C, Gonze X, Michenaud J-P. First-principles study of the stacking effect on the electronic properties of graphite(s). Carbon 1994;32(2):289-299.

23 It is clear that GGA (leading to a distance of $4.2 \AA$ and energy of $2-3 \mathrm{meV} /$ atom) seriously underestimates the interlayer binding energy and is in strong disagreement for the geometry. Thus, even if LDA is unable to describe the dispersion interactions, it is a better choice than the GGA as far as the geometry and energy differences are concerned.

24 Mounet N, Marzari N. First-principles determination of the structural, vibrational and thermodynamic properties of diamond, graphite, and derivatives. Phys. Rev. B 2005;71(20):205214.

25 Charlier J-C, Gonze X, Michenaud J-P, Graphite Interplanar Bonding: Electronic Delocalization and van der Waals Interaction. Europhys. Lett. 1994;28(6):403.

26 Dappe YJ, Basanta MA, Flores F, Ortega J. Weak chemical interaction and van der Waals forces between graphene layers: A combined density functional and intermolecular perturbation theory approach. Phys. Rev. B 2006;74(20):205434.

${ }^{27}$ Lewis JP, Glaesemann KR, Voth GA, Fritsch J, Demkov AA, Ortega J, et al. Further developments in the localorbital density-functional-theory tight-binding method. Phys. Rev. B 2001;64(19):195103.

28 Dappe YJ, Ortega J, Flores F. Intermolecular interaction in density functional theory: Application to carbon nanotubes and fullernes. Phys. Rev. B 2009;79(16):165409.

29 Born M, Huang K. Dynamical Theory of Crystal Lattices; Oxford University Press: New York, 1954.

${ }^{30}$ Fasolino A, Los JH, Katsnelson MI. Intrinsic ripples in graphene. Nature Mater. 2007;6:858-861.

31 Kaxiras E, Duesbery MS. Free energies of generalized stacking faults in Si and implications for the brittle-ductile transition. Phys. Rev. Lett. 1993;70(24):3752-3755.

32 Kolmogorov AN, Crespi VH. Registry-dependent interlayer potential for graphitic system. Phys. Rev. B
2005;71(23):235415.

33 Kolmogorov AN, Crespi VH. Smoothest Bearings: Interlayer Sliding in Multiwalled Carbon Nanotubes. Phys. Rev. Lett. 2000;85(22):4727-4730.

34 Kolmogorov AN, Crespi VH, Schleier-Smith MH, Ellenbogen JC. Nanotube-Substrate Interactions: Distinguishing Carbon Nanotubes by the Helical Angle. Phys. Rev. Lett. 2004;92(8):085503.

35 Scandolo S, Bernasconi M, Chiarotti GL, Focher P, Tosatti E. Pressure-Induced Transformation Path of Graphite to Diamond. Phys. Rev. Lett. 1995;74(20):4015-4018.

36 Suarez-Martinez I, Savini G, Haffenden G, Campanera J-M, Heggie MI. Dislocations of Burgers vector $\mathrm{c} / 2$ in graphite. Phys. Stat. Sol. (c) 2007;4(8):2958-2962.

37 Girifalco LA, Ladd RA, Energy of Cohesion, Compressibility, and the Potential Energy Functions of the Graphite System. J. Chem. Phys. 1956;25:693.

38 Benedict LX, Chopra NG, Cohen ML, Zettl A, Louie SG, Crespi VH. Microscopic determination of the interlayer binding energy in graphite. Chem. Phys. Lett. 1998;286(56):490-496.

39 Zacharia R, Ulbricht H, Hertel T. Interlayer cohesive energy of graphite from thermal desorption of polyaromatic hydrocarbons. Phys. Rev. B 2004;69(15):155406.

40 Grimsditch MH, Ramdas AK. Brillouin scattering in diamond. Phys. Rev. B 1975;11(8):3139-3148.

41 Schreiber E, Anderson OL, Soga N. Elastic Constants and Their Measurement. New York: McGraw-Hill; 1973.

42 Yagi T, Utsumi W, Yamakata M, Kikegawa T, Shimomura O. High-pressure in situ x-ray-diffraction study of the phase transformation from graphite to hexagonal diamond at room temperature. Phys. Rev. B 1992;46(10):6031-6039.

43 Hanfland M, Beister H, Syassen K. Graphite under pressure: Equation of state and first-order Raman modes. Phys. Rev. B 1989;39(17):12598-12603.

44 Zhao YX, Spain IL. X-ray diffraction data for graphite to 20 GPa. Phys. Rev. B 1989;40(2):993-997; to estimate $B_{a}$ they inadvertently used the planar bulk modulus instead of using the linear one (see Schreiber et al ${ }^{41}$ ).

45 Dienwiebel M, Verhoeven GS, Pradeep N, Frenken JWM, Heimberg JA, Zandbergen HW. Superlubricity of Graphite. Phys. Rev. Lett. 2004;92(12):126101.

46 Cumings J, Zettl A. Low-Friction Nanoscale Linear Bearing Realized from Multiwall Carbon Nanotubes. Science 2000;289(5479):602-604. 